\title{
Overexpression of LRIG1 regulates PTEN via MAPK/MEK signaling pathway in esophageal squamous cell carcinoma
}

\author{
XIAOFANG JIANG ${ }^{1}$ and HUIWU $\mathrm{LI}^{2,3}$ \\ ${ }^{1}$ Central Laboratory; ${ }^{2}$ Department of Biochemistry, School of Basic Medicine; ${ }^{3}$ Tumor Institute, \\ Affiliated Tumor Hospital, Xinjiang Medical University, Urumqi, Xinjiang 830011, P.R. China
}

Received April 8, 2016; Accepted June 20, 2016

DOI: $10.3892 / \mathrm{etm} .2016 .3606$

\begin{abstract}
The present study aimed to evaluate the role of leucine-rich repeats and immunoglobulin-like domain protein 1 (LRIGI) in the regulation of phosphatase and tensin homolog $(P T E N)$ expression in esophageal carcinogenesis. LRIG1 was overexpressed in esophageal squamous cell carcinoma (ESCC) cell lines, and the effect of LRIGI overexpression on the mRNA and protein expression levels of PTEN was evaluated by reverse transcription-quantitative polymerase chain reaction and western blotting. Furthermore, the effects of LRIGI overexpression on the cell cycle distribution and apoptosis of ESCC cells were examined by flow cytometry. Various cell signaling pathway inhibitors were used to assess the effects of LRIGl on downstream signaling in ESCC cell lines. In addition, the association between LRIGI and PTEN expression was examined in 48 samples from patients with ESCC. LRIG1 overexpression was demonstrated to downregulate PTEN expression in ESCC cell lines, and promote their proliferation and inhibit apoptosis. In addition, LRIG1-mediated suppression of PTEN expression was inhibited by the U0126 inhibitor, which suggests that LRIG1 may inhibit the activation of PTEN signaling molecules by triggering the mitogen-activated protein kinase (MAPK)/MAPK kinase 1 (MEK) signaling pathway. In conclusion, the present study demonstrated that overexpression of LRIGl significantly and adversely affected the survival of ESCC cells, and that the MAPK/MEK signaling pathway may be responsible for the repression of PTEN expression and function.
\end{abstract}

Correspondence to: Professor Huiwu Li, Tumor Institute, Affiliated Tumor Hospital, Xinjiang Medical University, 393 Xinyi Road, Urumqi, Xinjiang 830011, P.R. China

E-mail: huiwuli4321@sina.com

Key words: esophageal squamous cell carcinoma, leucine-rich repeats and immunoglobulin-like domain protein 1, phosphatase and tensin homolog, signaling pathway

\section{Introduction}

Leucine-rich repeats and immunoglobulin-like domain protein 1 (LRIGI) is located at chromosome 3p14, which is recurrently deleted in human cancers $(1,2)$. A previous study demonstrated that LRIG1 was a negative regulating factor of numerous oncogenic receptor tyrosine kinases (RTKs) (3). In two independent studies, LRIG1 was shown to downregulate the expression of four members of the epidermal growth factor (EGF) family of receptor tyrosine kinases (ERBB receptors), including the EGF receptor (EGFR), human epidermal growth factor receptor 2 (HER2), HER3 and HER4 $(3,4)$. In addition, LRIG1 was shown to reduce the levels of EGFR by accelerating receptor ubiquitylation and lysosomal degradation (4). Furthermore, LRIG1 has been reported to regulate ERBB receptor degradation and act as a tumor suppressor (5). However, at present, no correlation has been demonstrated between LRIG1 and EGFR expression in colorectal cancer (6). Thus, the various functions of $L R I G l$ in cancer remain uncertain.

Previous studies reported that LRIGl overexpression suppressed the growth of the PC3 prostate cancer cell line, and that this effect was overcome by androgen in patients with increased LRIG1 and androgen activities $(7,8)$. These findings suggested that LRIG1 may be unable to elicit sufficient tumor suppressive activity in a high-androgen environment. The prognostic significance of the LRIG1 protein in cervical cancer has been associated with tumor suppressors, oncogenes and numerous factors, including cancer subtype and stage (9). LRIG1 expression has been shown to be downregulated in a number of cancer types, although it was reported to be overexpressed in prostate and colorectal tumors $(7,8)$. The expression level and subcellular location of LRIG proteins have a prognostic value in brain tumors (10). Due to this heterogeneity in results, whether the function of LRIG1 suppresses or promotes tumor growth remains unclear.

Phosphatase and tensin homolog (PTEN) is a type I protein tyrosine phosphatase containing five domains. The phosphatase and tensin domains of PTEN are recurrently deleted from chromosome 10q23 in oncogenic events (11). PTEN targets a wide range of molecules, thereby regulating tumorigenic functions such as apoptosis, the cell cycle, cell adhesion and cell migration (12). The function of LRIG1 and 
its regulatory association with the PTEN gene in esophageal squamous cell carcinoma (ESCC) remains unclear. Therefore, the present study aimed to investigate the association between LRIGl and PTEN in ESCC cell lines and patient samples.

\section{Materials and methods}

Reagents. The pEGFP-N1-LRIG1 plasmid was provided by Professor Guo Dongsheng (Huazhong University of Science \& Technology, Wuhan, China). The empty pEGFP-N1 vector, used as a control, was provided by Xinjiang Medical University (Urumqi, China). Inhibitors of phosphoinositide 3-kinase (PI3K; LY294002), mitogen-activated protein kinase (MAPK) kinase 1 (MEK; PD98059, U0126), p38-MAPK2 (SB203580), janus kinase 2 (JAK2; AG-490), $\mathrm{Ca}^{2+} / \mathrm{calmodulin}-d e p e n d e n t$ protein kinase II $(\mathrm{KN}-62)$ and calcineurin (FK-506) were purchased from Sigma-Aldrich (St. Louis, MO, USA). The protein kinase C (PKC) inhibitor, bisindolylmaleimide I,was obtained from Cell Signaling Technology, Inc. (Danvers, MA, USA). Anti-LRIG1 antibody was purchased from Santa Cruz Biotechnology, Inc. (Dallas, TX, USA). Anti-PTEN antibody was purchased from Wuhan Boster Biological Technology, Ltd. (Wuhan, China).

Cell culture. Eca-109 human esophageal cancer cells were purchased from the Type Culture Collection of the Chinese Academy of Sciences (Shanghai, China). KYSE-450 human esophageal cancer cells were purchased from the Beijing Institute for Cancer Research (Beijing Cancer Hospital, Beijing, China). All cells were maintained according to the manufacturer's protocols. Briefly, the cells were cultured at a density of $4 \times 10^{4}$ in Roswell Park Memorial Institute-1640 medium (Gibco; Thermo Fisher Scientific, Inc., Waltham, MA, USA) supplemented with 5\% fetal bovine serum (Hangzhou Sijiqing Biological Engineering Materials Co., Ltd., Hangzhou, China) and $100 \mu / \mathrm{ml}$ penicillin/streptomycin in six-well plates in a $5 \% \mathrm{CO}_{2}$ humidified incubator at $37^{\circ} \mathrm{C}$. After achieving $80 \%$ confluence, the Eca-109, and KYSE-450 cells were pretreated with LY294002 (10 $\mu \mathrm{M})$, PD98059 $(50 \mu \mathrm{M})$, U0126 $(10 \mu \mathrm{M})$, bisindolylmaleimide I $(4 \mu \mathrm{M})$, SB203580 $(10 \mu \mathrm{M})$, AG-490 $(10 \mu \mathrm{M}), \mathrm{KN}-62(10 \mu \mathrm{M})$ and FK-506 $(10 \mu \mathrm{M})$ for $1 \mathrm{~h}$ at $37^{\circ} \mathrm{C}$, followed by transfection with pEGFP-N1-LRIG1 or pEGFP-N1 (control) plasmids using Lipofectamine 2000 (Invitrogen; Thermo Fisher Scientific, Inc.) for $48 \mathrm{~h}$.

Patients. A total of 48 pairs of ESCC and nearby normal esophageal tissue samples were obtained from Hazak patients at the Affiliated Tumor Hospital of Xinjiang Medical University between January 2008 and June 2009. Informed consent was obtained from all patients, and the present study was approved by the research ethical committee of the Affiliated Tumor Hospital of Xinjiang Medical University. All patients underwent a radical esophagectomy without preoperative chemotherapy or radiotherapy, and the pathological diagnosis of ESCC was confirmed by histopathological analyses. Tumors were classified as stage I $(n=1)$, IIa $(\mathrm{n}=13)$, IIb $(n=5)$ or III $(n=29)$, according to the criteria of the Unio Internationale Contra Cancrum tumor-node-metastasis classification system (13) of malignant tumors. Tumors and coupled normal esophageal tissues from the same patient were harvested and analyzed by senior pathologists, followed by freezing in liquid nitrogen within $30 \mathrm{~min}$ postoperatively and storing at $-80^{\circ} \mathrm{C}$ until experimental use.

Immunohistochemical analysis. Cells $\left(4 \times 10^{4}\right)$ were cultured in RPMI-1640 supplemented with 5\% fetal bovine serum and $100 \mu / \mathrm{ml}$ penicillin/streptomycin in six-well plates. The plates were then placed in a $5 \% \mathrm{CO}_{2}$ humidified incubator at $37^{\circ} \mathrm{C}$. Following $24 \mathrm{~h}$ of growth, cells attached to the glass slide were fixed with $4 \%$ poly formaldehyde and counted by immunohistochemical staining. Further steps were conducted in a hydrated chamber at room temperature. Endogenous peroxidase activity was inhibited using a peroxidase block (Wuhan Boster Biological Technology, Ltd.) for 5 min, after which the slides were incubated with $20 \%$ normal goat serum in $50 \mathrm{mM}$ Tris- $\mathrm{HCl}(\mathrm{pH}$ 7.4). The cells attached to the glass slide were incubated with goat anti-human LRIG1 monoclonal antibody $(1: 100)$ overnight at $4^{\circ} \mathrm{C}$. Subsequently, the cells were incubated with rabbit horseradish peroxidase (HRP)-conjugated anti-goat IgG secondary antibody (1:6,000; cat. no. BA1060; Wuhan Boster Biological Technology,Ltd.) at $37^{\circ} \mathrm{C}$ for $30 \mathrm{~min}$, following washing with phosphate-buffered saline (PBS), and stained with 3,3'-diaminobenzidine. The slides were visualized by fluorescent inverted microscopy.

Reverse transcription-quantitative polymerase chain reaction $(R T-q P C R)$. Total RNA was extracted from the tissue samples and ESCC cell lines using TRIzol reagent (Invitrogen; Thermo Fisher Scientific, Inc.), according to the manufacturer's protocol. cDNA was synthesized from $1 \mu \mathrm{g}$ RNA in a $20 \mu 1$ reaction mixture using the Reverse Transcription System (cat. no. A3500; Promega Corporation, Madison, WI, USA).cDNA was amplified using the following primers: GAPDH forward, 5'-GACCTGACCTGCCGC CTA-3' and reverse, 5'-AGGAGTGGGTGTCGCTGT-3'; LRIG1 forward, 5'-ACCTGTGAACCTTGGAGGAAT-3' and reverse, 5'-TCATCGCACACGAAACTCTC-3'; and PTEN forward, 5'-AAAGGGACGAACTGTGGTGTAATG-3' and reverse, 5'-TGGTCCTTACTTCCCCATAGAA-3'. qPCR was performed using the SYBR Green PCR Master Mix (Invitrogen; Thermo Fisher Scientific, Inc.), according to the manufacturer's protocol. PCR was performed in a reaction volume of $20 \mu \mathrm{l}$ containing $10 \mu \mathrm{l} 2 \mathrm{X}$ PCR Master, $2 \mu \mathrm{l} \mathrm{cDNA}$, $7 \mu \mathrm{l}$ water, $0.5 \mu \mathrm{l}$ forward primer and $0.5 \mu \mathrm{l}$ reverse primer. The thermal cycling conditions were as follows: $5 \mathrm{~min}$ at $95^{\circ} \mathrm{C}$, followed by 40 cycles $\left(94^{\circ} \mathrm{C}, 30 \mathrm{sec} ; 60^{\circ} \mathrm{C}, 30 \mathrm{sec} ; 72^{\circ} \mathrm{C}\right.$, $30 \mathrm{sec}$ ). Relative mRNA expression levels were determined by normalization to GAPDH using the $2^{-\triangle \Delta \mathrm{Cq}}$ method (14).

Western blot analysis. Cells and tissues (following tissue homogenization) were lysed using cell lysis buffer $(5 \mathrm{mM}$ Tris- $\mathrm{HCl}, 1 \mathrm{mM}$ EGTA, $1 \mathrm{mM}$ EDTA, $150 \mathrm{mM} \mathrm{NaCl}, 1 \%$ NP-40, 0.1\% SDS and $1 \mathrm{mM}$ PMSF; pH 7.5) containing $1 \%$ Triton X-100 with protease inhibitors at $4^{\circ} \mathrm{C}$ for $30 \mathrm{~min}$. Protein lysates were purified by centrifugation $(15,000 \mathrm{x} \mathrm{g}$; $\left.4^{\circ} \mathrm{C} ; 10 \mathrm{~min}\right)$, after which equal quantities of protein $(60 \mu \mathrm{g})$ were separated from various cells and tissue samples and boiled for $5 \mathrm{~min}$. The proteins were then separated by $10 \%$ SDS-PAGE and transferred onto nitrocellulose membranes. 
The membranes were blocked overnight at $4^{\circ} \mathrm{C}$ using blocking solution containing $1 \%$ bovine serum albumin (Sangon Biotech Co., Ltd., Shanghai, China), 5\% skimmed milk and $0.05 \%$ Tween-20. Subsequently, the membranes were incubated overnight at $4^{\circ} \mathrm{C}$ with polyclonal goat anti-human LRIG1 (1:250; cat. no. SC-134435; Santa Cruz Biotechnology, Inc.), monoclonal rabbit anti-human PTEN (1:100; cat. no. BA1377; Wuhan Boster Biotechnology, Co., Ltd.), and monoclonal rabbit anti-human GAPDH (1:400; cat. no. BM1623; Wuhan Boster Biotechnology, Co., Ltd.) antibodies, followed by washing three times with Tris-buffered saline supplemented with Tween-20 and incubation overnight at $4{ }^{\circ} \mathrm{C}$ with monoclonal HRP-conjugated goat anti-rabbit (cat. no. BA1055; Wuhan Boster Biotechnology Co., Ltd.) and rabbit anti-goat (cat. no. BA1060; Wuhan Boster Biotechnology Co., Ltd.) secondary antibodies (1:6,000). The protein complexes were evaluated using an enhanced chemiluminescence system (Wuhan Boster Biological Technology, Ltd.), and the blots were visualized using 3,3'-diaminobenzidine (Beijing Zhongshan Golden Bridge Biotechnology, Co., Ltd.). Western blot bands were quantified using Quantity One software version 4.6.2 (Bio-Rad Laboratories, Inc., Hercules, CA, USA), which measured the band intensity of each group. Band intensities were normalized to GAPDH as an internal control.

Apoptosis analysis. The level of apoptosis was evaluated by Annexin V-fluorescein isothiocyanate (FITC) staining and flow cytometry. Following pretreatment with various inhibitors and transfection with pEGFP-N1-LRIG1 plasmids, the cells were harvested, washed with pre-chilled PBS and resuspended in $1 \mathrm{X}$ binding buffer at a density of $\sim 1 \times 10^{6}$ cells $/ \mathrm{ml}$. Subsequently, $\sim 100 \mu \mathrm{l}$ solution was mixed with $5 \mu \mathrm{l}$ Annexin V-FITC and $5 \mu \mathrm{l}$ propidium iodide (PI) for $15 \mathrm{~min}$, followed by addition of $400 \mu \mathrm{l}$ $1 \mathrm{X}$ binding buffer. Apoptosis was assessed within $1 \mathrm{~h}$ using the BD FACSCalibur (BD Biosciences, San Jose, CA, USA). The percentage of apoptotic cells was assessed using CXP cytometer software version 2.0 (Beckman Coulter, Inc., Brea, CA, USA).

Cell cycle distribution analysis. Cells were cultured in six-well plates until reaching $\sim 60-70 \%$ confluence, followed by transfection with pEGFP-N1-LRIG1 plasmid. After $48 \mathrm{~h}$, the cells were harvested using $0.25 \%$ trypsin, washed with $1 \mathrm{X}$ PBS and centrifuged $(1,500 \mathrm{x} \mathrm{g} ; 5 \mathrm{~min})$ at $4^{\circ} \mathrm{C}$. The resulting cell pellets were resuspended in Eca-109 and KYSE-450 cells and fixed with $75 \%$ ethanol for $1 \mathrm{~h}$ at $4^{\circ} \mathrm{C}$, followed by washing with PBS, resuspending in $0.05 \mathrm{mg} / \mathrm{ml}$ PI solution containing RNase A and incubation in the dark at $4^{\circ} \mathrm{C}$ for $30 \mathrm{~min}$. Subsequently, the cell cycle distribution was analyzed using a flow cytometer.

Statistical analysis. Standard two-tailed t-tests were performed on two data sets. Representative data from three experiments are presented, in which bars represent the mean \pm standard deviation. Summary statistics of patient data were calculated to demonstrate patient characteristics. Fisher's exact test was used to evaluate the association between two categorical variables. SPSS 17.0 software (SPSS, Inc., Chicago, IL, USA) was used to perform Fisher's exact test and t-tests to analyze the differences in gene expression between the ESCC and non-cancerous mucosa. $\mathrm{P} \leq 0.05$ was considered to indicate a statistically significant difference.

\section{Results}

LRIG1 downregulates PTEN expression in esophageal cancer cell lines. The pEGFP-N1-LRIG1 plasmid was transfected into Eca-109 and KYSE-450 cell lines, in which LRIG1 expression levels were intrinsically low. LRIG1 expression was inversely correlated with PTEN expression in Eca-109 and KYSE-450 cell lines following transfection of the cells. At $48 \mathrm{~h}$ after transfection, fluorescence microscopy detected green fluorescence in the transfected cells (Fig. 1A and B). The mRNA expression levels of LRIGl were examined by RT-qPCR at $48 \mathrm{~h}$ following transfection, and demonstrated a significant increase (magnitude, $>2^{12}$ ) in the expression levels of LRIGI mRNA in the transfected cells, as compared with the negative controls (Fig. 1C). In addition, western blotting demonstrated that the protein expression levels of LRIG1 were significantly increased by $\sim 10.1 \%$ in KYSE-450 cells and 50.5\% in Eca-109 cells transfected with pEGFP-N1-LRIG1 plasmid, as compared with the negative controls ( $\mathrm{P}<0.05$; Fig. 1D).

RT-qPCR and western blotting demonstrated a correlation between PTEN and LRIG1 expression levels in Eca-109 and KYSE-450 cell lines following transfection with the pLRIG1-GFP-N1 plasmid (Fig. 1C and D). Upregulation of LRIG1 expression markedly decreased the mRNA and significantly decreased the protein expression levels $(\mathrm{P}<0.05)$ of PTEN in the transfected cells (Fig. 1C and D), as compared with the negative controls.

The cells were pretreated with various inhibitors to elucidate the signal transduction pathways involved in the LRIG1-mediated inhibition of PTEN expression. The ability of LRIG1 to downregulate PTEN expression was diminished by pretreatment of Eca-109 and KYSE-450 cells with U0126 and KN-62 inhibitors (Fig. 2). These results suggest that LRIG1-induced downregulation of PTEN in esophageal cancer cells involves the MEK signaling pathway.

Overexpression of LRIG1 reduces apoptosis in Eca-109 and KYSE-450 cells. The effect of LRIG1 overexpression on the apoptosis of Eca-109 and KYSE-450 cells transfected with the pEGFP-N1-LRIG1 plasmid was assessed by flow cytometry. The percentage of apoptotic Eca-109 cells was significantly decreased from $51.10 \%$ in control cells transfected with pEGFP-N1 plasmid to $26.76 \%$ in Eca-109 cells transfected with pEGFP-N1-LRIG1 plasmid ( $\mathrm{P}<0.05$; Fig. 3). Similarly, the percentage of apoptotic KYSE-450 cells was significantly decreased from $41.70 \%$ in control cells to $19.47 \%$ in $L R I G 1$ overexpressing cells $(\mathrm{P}<0.05$; Fig. 3$)$.

LRIG1 overexpression arrests the cell cycle in the S-phase. The effects of LRIG1 overexpression on the cell cycle distribution of Eca-109 and KYSE-450 cells was assessed by flow cytometry (Fig. 4A-C). As compared with the control cells, LRIG1 overexpression increased the percentage of Eca-109 and KYSE-450 cells in the S-phase of the cell cycle. The percentage of Eca-109 cells in the S-phase was increased from $42.11 \%$ of the control cells to $47.19 \%$ of the LRIG1 overexpressing cells (Fig. 4D). Similarly, the percentage of KYSE-450 cells in the S-phase was increased from $58.64 \%$ of the control cells to $71.24 \%$ of the LRIGl overexpression cells (Fig. 4D). 
A

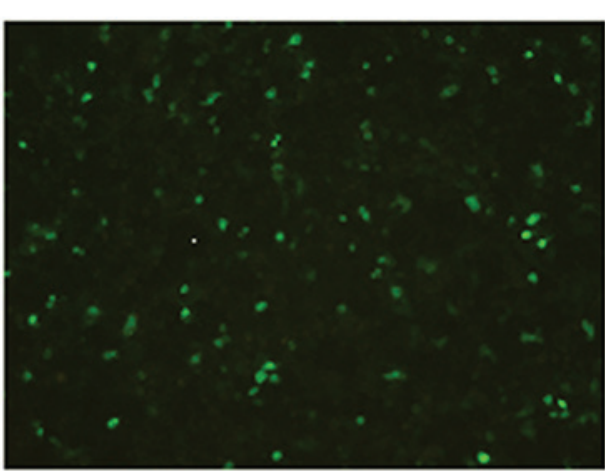

C

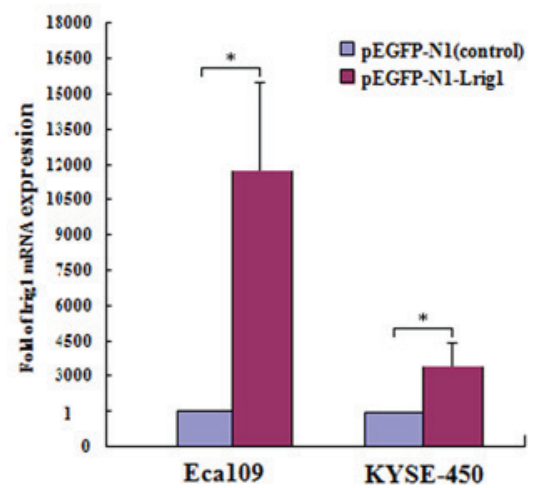

D
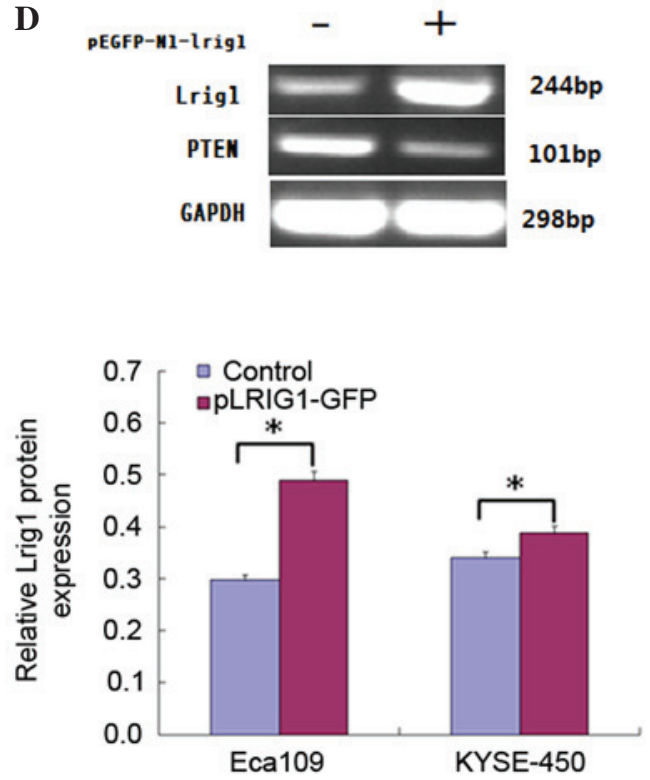

B
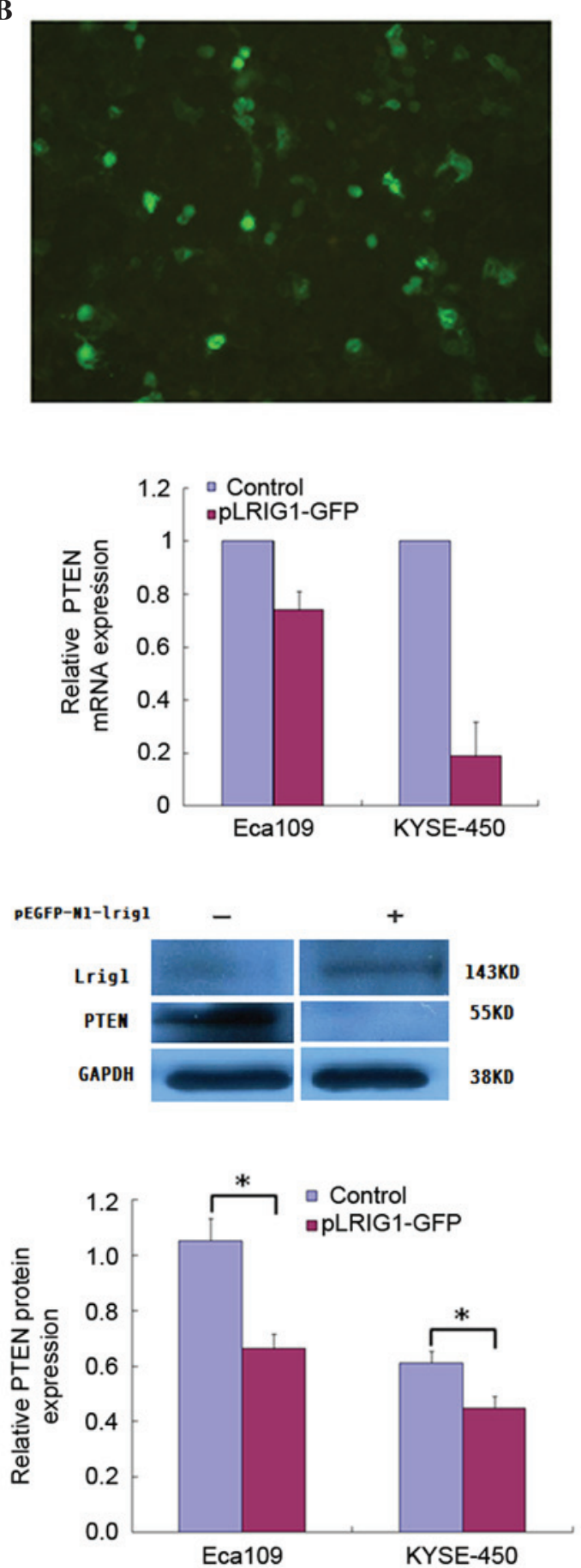

Figure 1. LRIG1 overexpression inhibits PTEN expression in esophageal squamous cell carcinoma cell lines. (A) Eca-109 (magnification, x50) and (B) KYSE-450 cells (magnification, x100) were transfected with pLRIG1-GFP or pEGFP-N1 (control) for 48 h, after which the cells were observed under a microscope. (C) The mRNA expression levels of LRIG1 and PTEN in Eca-109 and KYSE-450 cells were analyzed by reverse transcription-quantitative polymerase chain reaction. The mRNA expression levels of LRIG1 were increased 11,680-fold (Eca-109) and 3,351 (KYSE-450) compared with the negative control. (D) The protein expression levels of LRIG1 and PTEN in Eca-109 and KYSE-450 cells were determined by western blotting and densitometry. The protein expression levels of LRIG1 were increased by $\sim 10.1 \%$ in KYSE-450 cells and $50.5 \%$ in Eca-109 cells compared with the negative control cells. The mRNA and protein expression levels of PTEN

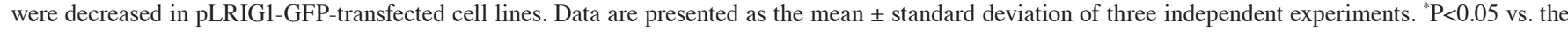
control. LRIG1, leucine-rich repeats and immunoglobulin-like domain protein 1; PTEN, phosphatase and tensin homolog; GFP, green fluorescent protein.

LRIG1 protein localization in Eca-109 and KYSE-450 cells. The localization of LRIG1 in Eca-109 and KYSE-450 cells was determined by immunohistochemical analyses. The perinuclear staining of LRIG1 was observed by microscopy in Eca-109 cells following transfection of the cells with the pLRIG1-GFP plasmid. In addition, the LRIG1 protein was primarily observed in the cytoplasm of KYSE-450 cells (Fig. 5).

PTEN mRNA expression negatively correlated with LRIGI in cancerous tissue. Total RNA was isolated from ESCC tissues and corresponding normal distant tissues from the 
Table I. Association between LRIGI and PTEN mRNA expression in esophageal squamous cell carcinoma cancer tissues and matched normal tissues $(\mathrm{n}=48)$.

\begin{tabular}{|c|c|c|c|c|c|c|c|c|}
\hline \multirow[b]{2}{*}{ Gene } & \multicolumn{2}{|c|}{ LRIGI (tumor) } & \multirow[b]{2}{*}{$R$} & \multirow[b]{2}{*}{ P-value } & \multicolumn{2}{|c|}{ LRIG1 (normal) } & \multirow[b]{2}{*}{$R$} & \multirow[b]{2}{*}{ P-value } \\
\hline & + & - & & & + & - & & \\
\hline PTEN & & & 0.478 & 0.002 & & & 0.181 & 0.183 \\
\hline+ & 29 & 9 & & & 18 & 19 & & \\
\hline- & 2 & 8 & & & 3 & 8 & & \\
\hline
\end{tabular}

LRIG1, leucine-rich repeats and immunoglobulin-like domain protein 1; PTEN, phosphatase and tensin homolog.
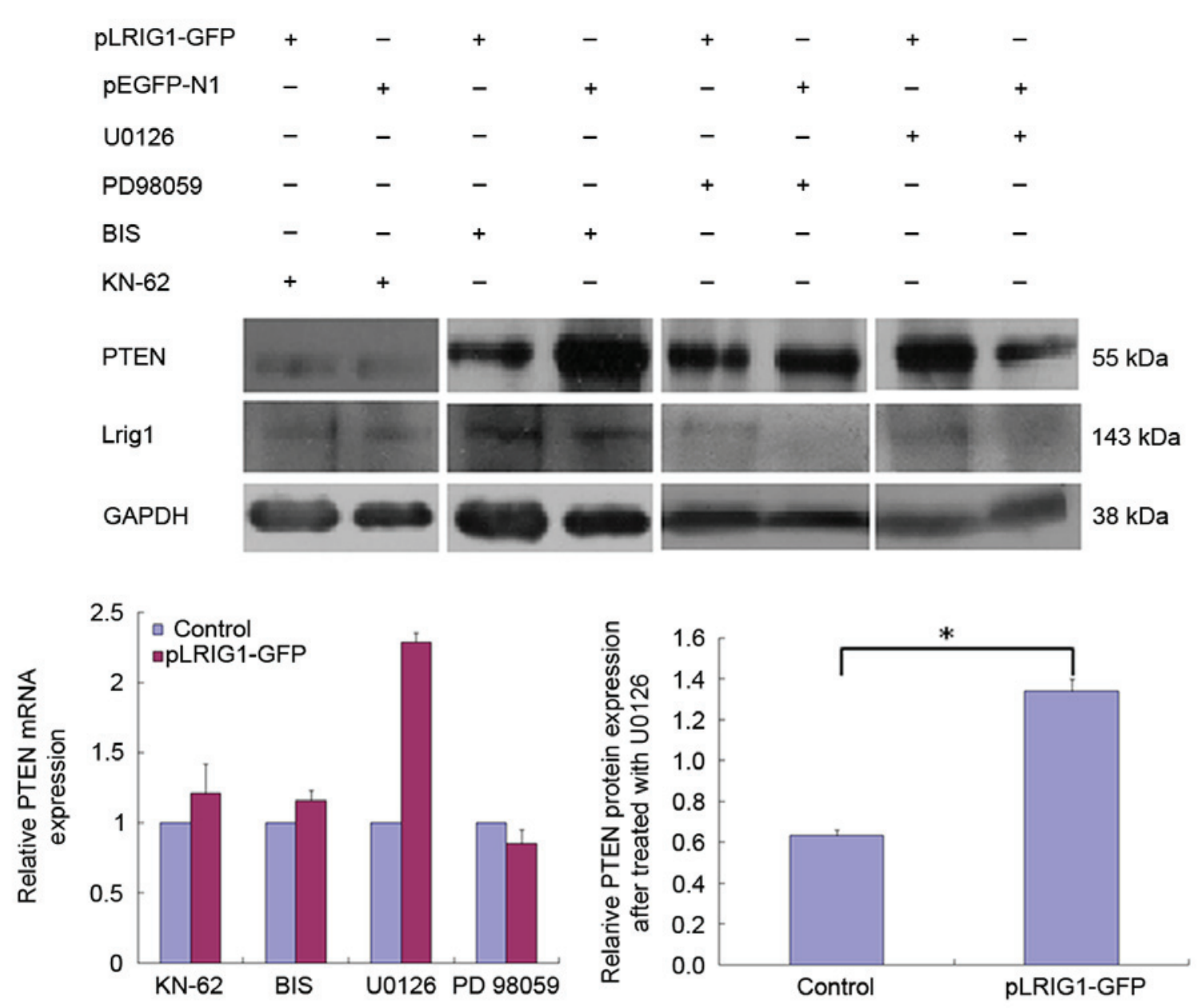

Figure 2. LRIG1-mediated inhibition of PTEN expression in esophageal squamous cell carcinoma cell lines was attenuated by the U0126 inhibitor. Eca-109 and KYSE-450 cells were pretreated with various signaling pathway inhibitors for $1 \mathrm{~h}$, followed by transfection with pLRIG1-GFP. LRIG1-mediated downregulation of PTEN mRNA expression was diminished by pretreatment with $10 \mu \mathrm{M} \mathrm{KN}-62,4 \mu \mathrm{M}$ BIS, $10 \mu \mathrm{M}$ PD98059 and $10 \mu \mathrm{M}$ U0126. LRIG-mediated downregulation of PTEN protein expression was significantly diminished by U0126. Data are presented as the mean \pm standard deviation of three independent experiments. "P<0.05 vs. the control. LRIG1, leucine-rich repeats and immunoglobulin-like domain protein 1; PTEN, phosphatase and tensin homolog; GFP, green fluorescent protein; BIS, bis-indolylmaleimide I.

same patient, and the mRNA expression levels of $L R I G 1$ and $P T E N$ were determined by RT-qPCR. The mRNA expression levels of PTEN were inversely correlated to the expression levels of $L R I G 1$ in the cancerous tissue $(R=0.478 ; \mathrm{P}=0.002$; Table I). No significant correlation was observed between clinical pathological factors and the expression levels of LRIG1 or PTEN.

\section{Discussion}

LRIG1, a membrane-associated protein, has been shown to inhibit growth factor signal transduction from oncogenic RTKs, including EGFR, and MET and RET proto-oncogenes (15-17). The downregulation of LRIGl expression results in spontaneous tumor formation, which suggests that 
A

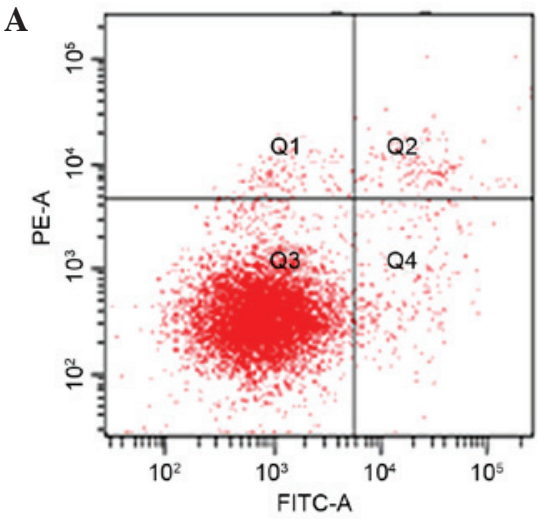

C

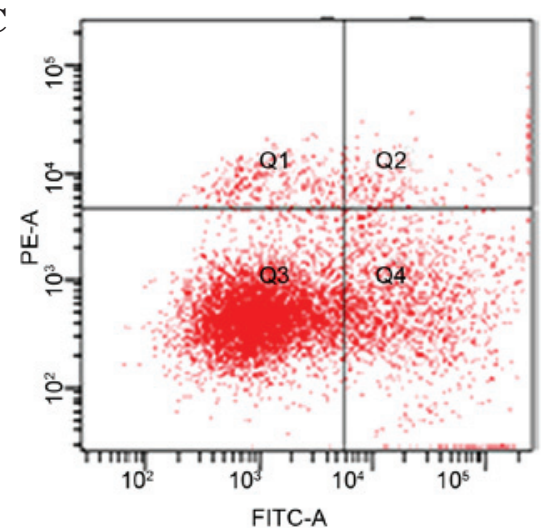

B

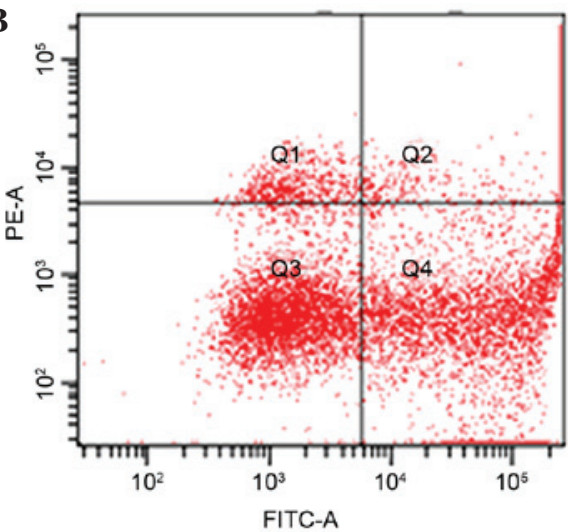

D

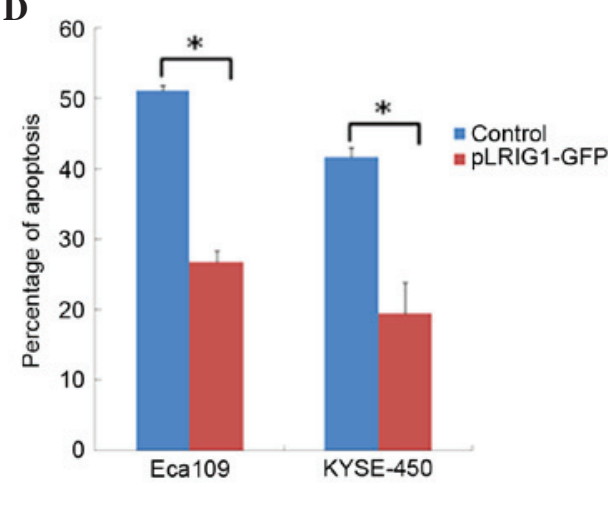

Figure 3. LRIG1 reduced the apoptosis of esophageal squamous cell carcinoma cell lines. Eca-109 and KYSE-450 cells were transfected with pLRIG1-GFP for $48 \mathrm{~h}$, after which they were double-stained with Annexin V/FITC and propidium iodide and analyzed by flow cytometry. The gate setting distinguished between viable (Q3), necrotic (Q1), early apoptotic (Q4) and late apoptotic (Q2) cells. (A) Untransfected control cells, (B) transfected with pEGFP-N1 control cells and (C) Eca-109 cells transfected with pLRIG1-GFP. (D) Cell apoptosis was significantly reduced by LRIG1 overexpression in Eca-109 and KYSE-450 cell lines. Data are presented as the mean \pm standard deviation. ${ }^{*} \mathrm{P}<0.05$ vs. the control. LRIG1, leucine-rich repeats and immunoglobulin-like domain protein 1; GFP, green fluorescent protein; FITC, fluorescein isothiocyanate; PE, phycoerythrin.

LRIG1 may serve as a tumor suppressor in certain types of cancer (2). However, LRIGl has been shown to be overexpressed in numerous cancers, including leukemia, astrocytoma and prostate cancers, thus suggesting that LRIGI may perform general downregulation in all types of human tumors $(7,18)$, and that the role of LRIGl may be masked by other factors. Therefore, the expression and function of LRIGl should be carefully evaluated. In a previous study performed by the authors, it was demonstrated that LRIG1 expression was not associated with EGFR expression, although it was correlated with HER2 expression in ESCC (19), which was consistent with the demonstrated correlation between increased gene copy numbers of HER2 and LRIG1 in a previous study (20).

The progression of esophageal cancer has been associated with the loss of tumor suppressor genes, including PTEN (21). PTEN may be an important biological marker in the treatment of human esophageal cancer (21). The present study demonstrated a significant correlation between LRIGI and PTEN mRNA expression levels in ESCC cell lines, and in tumor samples from patients with ESCC. Notably, LRIGI overexpression downregulated PTEN expression in Eca-109 and KYSE-450 cell lines, indicating that LRIG1 may have an oncogenic role. The expression and subcellular localization of LRIG1 may be associated with specific clinicopathological features of ependymoma tumors, and thus may be of great importance in carcinogenesis (22). In human oligodendroglioma, the cytoplasmic and perinuclear localizations of
LRIG1 were associated with the expression of various genes; thus suggesting that LRIG1 may perform various functions. In the present study, immunohistochemical analysis demonstrated that LRIG1 was predominantly expressed in the cytoplasm in ESCC cell lines. Notably, a number of perinuclear LRIG1 were observed following transfection with LRIG1 plasmid.

PTEN selectively inhibits the activation of extracellular signal-regulated kinase (ERK) in the MAPK signaling pathway $(23,24)$. The absence of PTEN in cancer cells has typically been associated with the increased activation of the PI3K/Akt signaling pathway, leading to malignant cancer transformation and progression $(25,26)$. In the present study, LRIG1-mediated downregulation of PTEN mRNA expression was diminished by pretreatment of ESCC cell lines with bisindolylmaleimide I (PKC inhibitor), PD98059 (ERK 1/2 inhibitor) and U0126 (MEK 1/2 inhibitor). These results suggest that LRIG1 regulates PTEN expression via the MAPK/MEK/ERK signaling pathway.

The apoptosis of Eca-109 and KYSE-450 cells was significantly inhibited following transfection of the cells with the pLRIG1-EGF plasmid, compared with the control cells. Furthermore, LRIG1 overexpression increased the percentage of Eca-109 and KYSE-450 cells in the S-phase of the cell cycle. These results suggested that LRIGl inhibited cell apoptosis and promoted the growth of cancer cells, serving as an oncogene in ESCC. In addition, these results 
A
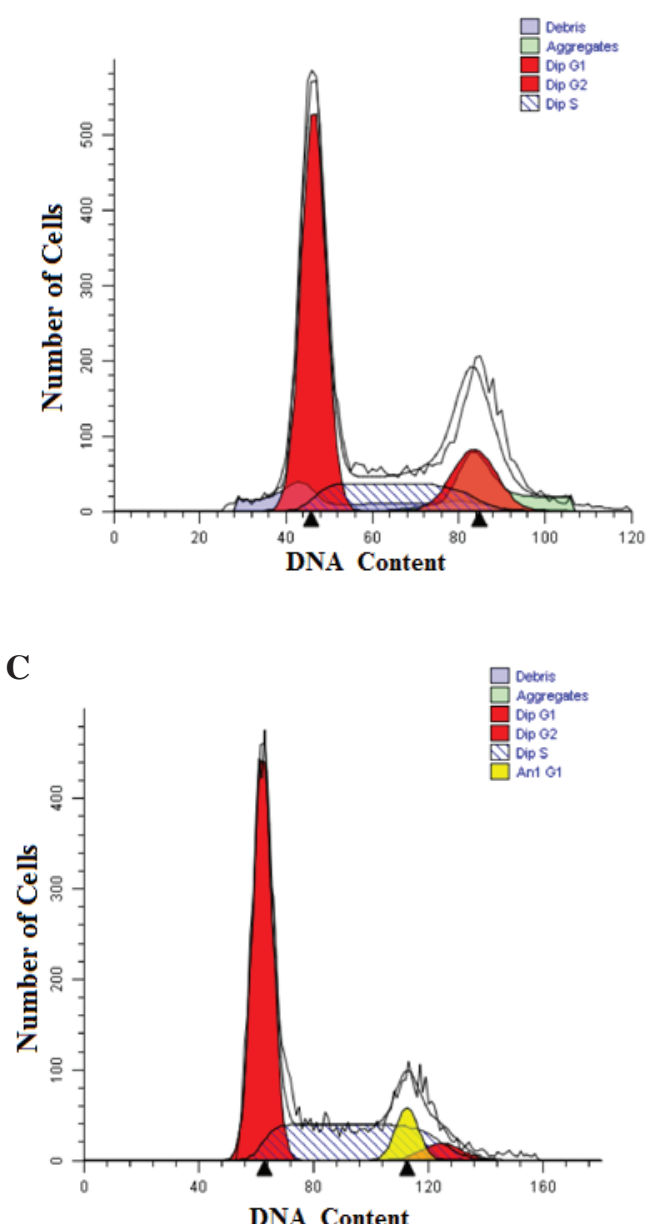

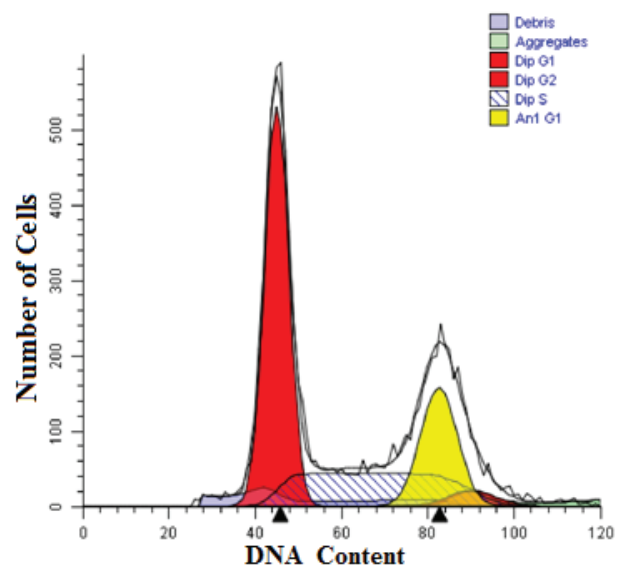

D

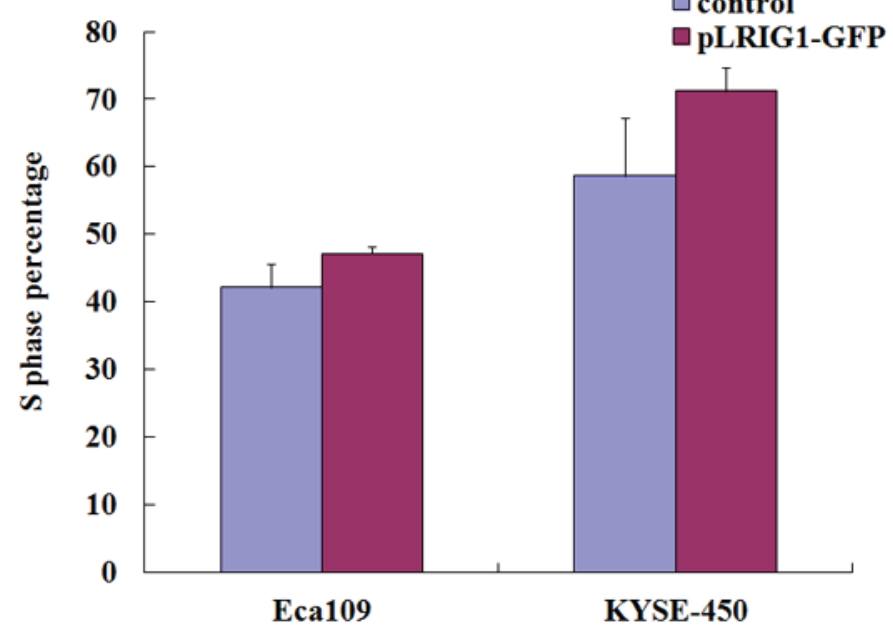

Figure 4. LRIG1 overexpression resulted in the accumulation of Eca-109 and KYSE-450 cells in the S phase. The cell cycle distribution of Eca-109 and KYSE-450 cells transfected with pLRIG1-GFP was determined by flow cytometry. (A-C) The value on the $x$-axis represents the DNA content and the shaded area indicates the percentage of cells in the S phase. (A) Untransfected control cells, (B) control cells transfected with pEGFP-N1 and (C) Eca-109 cells transfected with pLRIG1-GFP. (D) The percentages of Eca-109 and KYSE-450 cells in the S phase were increased following LRIG1 overexpression. Data are presented as the mean \pm standard deviation. LRIG1, leucine-rich repeats and immunoglobulin-like domain protein 1; GFP, green fluorescent protein; PE, phycoerythrin.
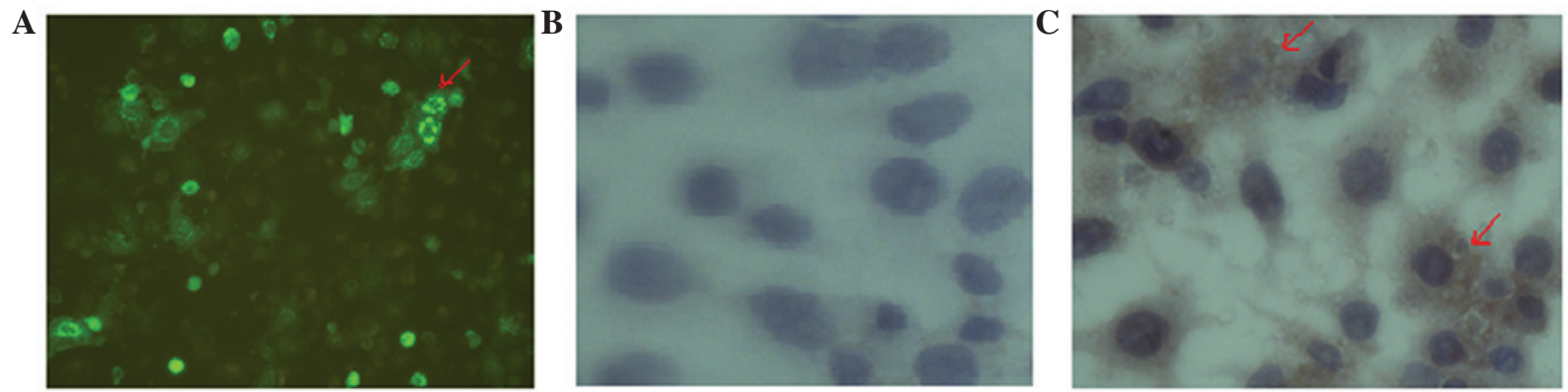

Figure 5. Immunohistochemical analysis was performed to determine the localization of leucine-rich repeats and immunoglobulin-like domain protein 1. (LRIG1) protein in KYSE-450 cells (magnification, x400). (A) pEGFP-N1-LRIG1 was successfully transfected into KYSE-450 cells and LRIG1 protein could be observed surrounding the nucleus. The red arrow indicates brown LRIG1 staining in the cytoplasm of KYSE-450 cells. (B) Low levels of LRIG1 protein were observed in the control cells. (C) Predominant LRIG1 staining was observed in the cytoplasm of KYSE-450 cells.

indicated that LRIG1 may function as an oncogene by regulating tumor-suppressor genes in a manner that is dependent on the subcellular localization of LRIG1. The overexpression of cytoplasmic LRIG1 in ESCC cell lines may have promoted cell growth by suppressing apoptosis and accelerating the cell cycle.
In conclusion, the present study demonstrated that LRIG1 overexpression downregulated PTEN expression in ESCC cell lines by activating the MAPK/MEK signaling pathway. In addition, LRIG1 was shown to function as a proto-oncogene by promoting tumor cell proliferation and simultaneously decreasing cellular apoptosis. LRIG1 
followed a non-canonical mechanism of interaction with various signal transduction pathways to elicit novel functions in the cytoplasm of tumor cells.

\section{Acknowledgements}

The present study was supported by the Nature Science Foundation of China (grant nos. 30950012 and 81360304) and the Xinjiang Endemic Molecular Biology Laboratory, China (grant no. xjdx0208-2011-02).

\section{References}

1. Nilsson J, Vallbo C, Guo D, Golovleva I, Hallberg B,Henriksson R and Hedman $\mathrm{H}$ : Cloning, characterization and expression of human LIG1. Biochem Biophys Res Commun 284: 31-37, 2001.

2. Hedman H, Nilsson J, Guo D and Henriksson R: Is LRIG1 a tumour suIs LRIG1 a tumour suppressor gene at chromosome 3p14.3? Acta Oncol 41: 352-354, 2002.

3. Laederich MB, Funes-Duran M, Yen L, Ingalla E, Wu X, Carraway KL IIIrd and Sweeney C: The leucine-rich repeat protein LRIG1 Is a negative regulator of ErbB family receptor tyrosine kinases. J Biol Chem 279: 47050-47056, 2004.

4. Gur G, Rubin C, Katz M, Amit I, Citri A, Nilsson J, Amariglio N, Henriksson R, Rechavi G, Hedman H, et al: LRIG1 restricts growth factor signaling by enhancing receptor ubiquitylation and degradation. EMBO J 23: 3270-3281, 2004.

5. Wang Y, Poulin EJ and Coffey RJ: LRIG1 is a triple threat: ERBB negative regulator, intestinal stem cell marker and tumour suppressor. Br J Cancer 108: 1765-1770, 2013.

6. Ljuslinder I, Golovleva I, Palmqvist R, Oberg A, Stenling R, Jonsson Y, Hedman H, Henriksson R and Malmer B: LRIG1 expression in colorectal cancer. Acta Oncol 46: 1118-112, 2007.

7. Thomasson M, Wang B, Hammarsten P, Dahlman A, Persson JL, Josefsson A, Stattin P, Granfors T, Egevad L, Henriksson R, et al: LRIG1 and the liar paradox in prostate cancer: A study of the expression and clinical significance of LRIG1 in prostate cancer. Int J Cancer 128: 2843-2852, 2010

8. Thomasson M, Hedman H, Ljungberg B and Henriksson R: Gene expression pattern of the epidermal growth factor receptor family and LRIG1 in renal cell carcinoma. BMC Res Notes 5: 216, 2012.

9. Lindström AK, Ekman K, Stendahl U, Tot T, Henriksson R, Hedman H and Hellberg D: LRIG1 and squamous epithelial uterine cervical cancer: Correlation to prognosis, other tumor markers, sex steroid hormones and smoking nternational. Int J Glynecol Cancer 18: 312-317, 2008.

10. Guo D, Nilsson J, Haapasalo H, Raheem O, Bergenheim T, Hedman H and Henriksson R: Perinuclear leucine-rich repeats and immunoglobulin-like domain proteins (LRIG1-3) as prognostic indicators in astrocytic tumors. Acta Neuropathol 111: 238-246, 2006.

11. Mak LH and Woscholski R: Targeting PTEN using small molecule inhibitors. Methods 77-78: 63-68, 2015
12. Zhang H, Sun $Z$ and Kong Y: Expression of PTEN, Her-2 and Glut-1 proteins in endometrial intraepithelial neoplasia and endometrioid. Xin Jiang Yi Ke Da Xue Xue Bao 34: 142-146, 2011.

13. Edge SB, Byrd DR, Compton CC, eds. AJCC Cancer Staging Manual, 7th edition. New York Springer p103-111, 2009.

14. Livak KJ and Schmittgen TD: Analysis of relative gene expression data using real-time quantitative PCR and the 2(-Delta Delta C(T)) Method. Methods 25: 402-408, 2001.

15. Lee JM, Kim B, Lee SB, Jeong Y, Oh YM, Song YJ, Jung S, Choi J, Lee S, Cheong KH, et al: Cbl-independent degradation of Met: Ways to avoid agonism of bivalent Met-targeting antibody. Oncogene 33: 34-43, 2014.

16. Goldoni S, Iozzo RA, Kay P, Campbell S, McQuillan A, Agnew C, Zhu JX, Keene DR, Reed CC and Iozzo RV: A soluble ectodomain of LRIG1 inhibits cancer cell growth by attenuating basal and ligand-dependent EGFR activity. Oncogene 26: 368-381, 2007.

17. Ledda F, Bieraugel O, Fard SS, Vilar M and Paratcha G: Lrig1 Is an endogenous inhibitor of Ret receptor tyrosine kinase activation, downstream signaling and biological responses to GDNF. J Neurosci 28: 39-49, 2008.

18. Thomasson M, Wang B, Hammarsten P, Dahlman A, Persson JL, Josefsson A, Stattin P, Granfors T, Egevad L, Henriksson R, et al: LRIG1 and the liar paradox in prostate cancer: A study of the expression and clinical significance of LRIG1 in prostate cancer. Int J Cancer 128: 2843-2852, 2011.

19. Jiang XF, Li H, Wang HJ, Li XM, Chen Y, Pang ZL, Chen HM and Li HW: Research of Lrig1 in esophageal squamous cell carcinoma. Chin J Dig 33: 123-124, 2013.

20. Ljuslinder I, Golovleva I, Henriksson R, Grankvist K, Malmer B and Hedman H: Co-incidental increase in gene copy number of ERBB2 and LRIG1 in breast cancer. Breast Cancer Res 11: 403, 2009.

21. Hou G, Lu Z, Liu M, Liu H and Xue L: Mutational analysis of the pten gene and its effects in esophageal squamous cell carcinoma. Dig Dis Sci 56: 1315-1322, 2011.

22. Yi W, Haapasalo H, Holmlund C, Järvelä S, Raheem $\mathrm{O}$, Bergenheim AT, Hedman $\mathrm{H}$ and Henriksson R: Expression of leucine-rich repeats and immunoglobulin-like domains (Lrig) proteins in human ependymoma relates to tumor location, who grade and patient age. Clin Neuropathol 28: 21-27, 2009.

23. Chappell WH, Steelman LS, Long JM, Kempf RC, Abrams SL, Franklin RA, Bäsecke J, Stivala F, Donia M, Fagone P, et al: Ras/Raf/MEK/ERK and PI3K/PTEN/Akt/mTOR inhibitors: Rationale and importance to inhibiting these pathways in human health. Oncotarget 2: 135-164, 2011.

24. Chetram MA and Hinton CV: PTEN regulation of ERK1/2 signaling in cancer. J Recept Signal Transduct Res 32: 190-195, 2012.

25. Maehama $T$ and Dixon JE: The tumor suppressor, PTEN/MMAC1, dephosphorylates the lipid second messenger, phosphatidylinositol 3,4,5-trisphosphate. J Biol Chem 273: 13375-13378, 1998.

26. Chetram MA, Don-Salu-Hewage AS and Hinton CV: ROS enhances CXCR4-mediated functions through inactivation of PTEN in prostate cancer cells. Biochem Biophys Res Commun 410: 195-200, 2011. 